\title{
Characterization of Sodium Channel Mutations in the Dengue Vector Mosquitoes Aedes aegypti and Aedes albopictus within the Context of Ongoing Wolbachia Releases in Kuala Lumpur, Malaysia
}

\author{
Noor Afizah Ahmad ${ }^{1, *} \mathbb{D}^{\text {, Nancy M. Endersby-Harshman }}{ }^{2, *}{ }^{\mathbb{D}}$, Nur Ruqqayah Mohd Mazni ${ }^{1}$, \\ Nur Zatil Aqmar Mohd Zabari ${ }^{1}$, Siti Nor Syazwani Amran ${ }^{1}$, \\ Muhammad Kamarul Ridhuan Ghazali ${ }^{1}$, Mohd Arif Abdul Karim ${ }^{1}$, Yoon Ling Cheong ${ }^{1}$ (), \\ Steven P. Sinkins ${ }^{3} \mathbb{D}$, Nazni Wasi Ahmad ${ }^{1}$ and Ary A. Hoffmann ${ }^{2} \mathbb{D}$ \\ 1 Medical Entomology Unit, Institute for Medical Research, Ministry of Health, Jalan Pahang, \\ Kuala Lumpur 50588, Malaysia; ruqqayahmazni@gmail.com (N.R.M.M.); \\ zatilaqmar27@gmail.com (N.Z.A.M.Z.); norsyazwaniamran@gmail.com (S.N.S.A.); \\ kamarulridhuan88@gmail.com (M.K.R.G.); mohdarif.pps@gmail.com (M.A.A.K.); \\ cheongyl@moh.gov.my (Y.L.C.); nazni@moh.gov.my (N.W.A.) \\ 2 PEARG, School of BioSciences, Bio21 Institute, The University of Melbourne, 30 Flemington Rd, Parkville, \\ Victoria 3010, Australia; ary@unimelb.edu.au \\ 3 Institute of Infection, Immunity and Inflammation, MRC-University of Glasgow Centre for Virus Research, \\ 464 Bearsden Road, Glasgow G61 1QH, UK; Steven.Sinkins@glasgow.ac.uk \\ * Correspondence: afizah.ahmad@moh.gov.my (N.A.A.); nancye@unimelb.edu.au (N.M.E.-H.)
}

Received: 17 July 2020; Accepted: 11 August 2020; Published: 13 August 2020

Simple Summary: Mosquitoes, Aedes aegypti and Ae. albopictus are vectors of dengue and must be controlled to prevent and contain outbreaks of this disease. Control by insecticide application is common and pyrethroid insecticides provide rapid knockdown of mosquitoes combined with relatively low mammalian toxicity. However, resistance to pyrethroids and other chemicals is causing problems for mosquito control around the world. In Malaysia, an alternative method of dengue reduction is employed which comprises releases of Ae. aegypti mosquitoes infected with a bacterium, Wolbachia, found naturally in other insects. Wolbachia turns the mosquitoes into incompetent vectors so they do not transmit the disease. Wolbachia mosquitoes are reared in the laboratory before release and must be able to survive in the field where they will encounter insecticides. Our study demonstrates benefits of crossing laboratory mosquitoes to those from the field over generations, so that the mosquito lines acquire field resistance characteristics (mutations in the sodium channel gene). We demonstrate that resistance mutations provide a survival advantage to Wolbachia Ae. aegypti mosquitoes, which must be maintained in laboratory lines by regular backcrossing. We also describe appearance of a sodium channel mutation in Malaysian Ae. albopictus which may indicate that pyrethroid resistance is increasing in this species.

Abstract: Specific sodium channel gene mutations confer target site resistance to pyrethroid insecticides in mosquitoes and other insects. In Aedes mosquito species, multiple mutations that contribute to resistance vary in their importance around the world. Here, we characterize voltage sensitive sodium channel $(V s s c)$ mutations in populations of Aedes aegypti from Kuala Lumpur, Malaysia, and look at their persistence in populations affected by ongoing Wolbachia releases (a dengue control measure). We also describe a Vssc mutation in Aedes albopictus (F1534L) found for the first time in Malaysia. We show that there are three predominant Vssc haplotypes in Aedes aegypti in this region, which all persist with regular backcrossing, thereby maintaining the original genetic composition of the populations. We identify changes in genotype frequency in closed populations of Ae. aegypti maintained for multiple generations in laboratory culture, suggesting different fitness 
costs associated with the genotypes, some of which may be associated with the sex of the mosquito. Following population replacement of Ae. aegypti by Wolbachia in the target area, however, we find that the VSSC mutations have persisted at pre-release levels. Mosquitoes in two genotype classes demonstrate a type I pyrethroid resistance advantage over wildtype mosquitoes when exposed to $0.25 \%$ permethrin. This resistance advantage is even more pronounced with a type II pyrethroid, deltamethrin $(0.03 \%)$. The results point to the importance of these mutations in pyrethroid resistance in mosquito populations and the need for regular backcrossing with male mosquitoes from the field to maintain similarity of genetic background and population integrity during Wolbachia releases.

Keywords: pyrethroid resistance; target site; $k d r$

\section{Introduction}

Dengue is a mosquito-borne infection that has become a major international public health concern. Malaysia is one of the most affected countries, with a total of 130,101 dengue cases and 182 deaths being recorded for 2019. This reflects an increase of $61.4 \%$ in terms of dengue cases and $23.8 \%$ in terms of deaths as compared to a similar period in 2018 [1]. In Malaysia, reducing human cases of dengue still relies on the control of vector mosquitoes, through fogging using permethrin, deltamethrin and malathion to kill adult mosquitoes, and larviciding using temephos to kill larvae. However, problems with insecticide resistance have arisen [2-4] due to a strong selective pressure from frequent application of insecticides which eventually will threaten the continued success of current vector control interventions.

In Malaysia, selection for insecticide resistance in Aedes species likely began with fogging operations using malathion which has continued since the early 1970s, and fogging with permethrin which has occurred since early 1996 [5]. Regular indoor residual spraying with dichlorodiphenyltrichloroethane (DDT) was also undertaken in Malaysia for 26 years, which then switched to deltamethrin in 1997 [6]. Pyrethroid insecticides are still widely used to control mosquito vector populations $[7,8]$, not only by the Ministry of Health, but also by private companies and communities to control mosquitoes as well as other household pests [9]. The versatility of pyrethroids which can be applied in different forms and in a range of situations has made this group the most intensively used insecticide for vector control against dengue in Kuala Lumpur.

Wan Norafikah et al. [3] found permethrin resistance in Aedes aegypti from Kuala Lumpur and identified a metabolic resistance component. Ishak et al. [4] found highest levels of pyrethroid resistance in Ae. aegypti and Ae. albopictus from Kuala Lumpur when compared to mosquitoes from Penang, Johor Bharu, and Kota Bharu. Aedes albopictus remained susceptible to pyrethroid insecticides except in Kuala Lumpur, where it showed moderate resistance to a type I and a type II pyrethroid [4]. The presence of permethrin (type I pyrethroid) resistance in field-collected Ae. aegypti from several urban areas in Kuala Lumpur has been reported, with the resistance ratio ranging from 4.28 to 4.47-fold, whilst for larvae, the resistance ratio ranged from 5.29 to 5.57-fold (commercial grade, 10.9\% a. i. $w / v$ ) [3]. Loke et al. [2] reported temporal variation in susceptibility to insecticides in a dengue endemic area in Shah Alam, with organophosphates being the most toxic to Ae. aegypti among the four classes of insecticides tested overall.

Pyrethroid resistance is a major concern in Malaysia and two mechanisms of resistance (target-site resistance (also known as knockdown resistance, $k d r$ ) and metabolic resistance) have been identified in Ae. aegypti $[4,10,11]$, while metabolic detoxification and possibly reduced cuticular penetration have been implicated in resistance in Ae. albopictus [12]. Although metabolic resistance is an important mechanism of pyrethroid resistance in Ae. aegypti in Malaysia, target-site resistance involving the voltage-sensitive sodium channel (VSSC) appears particularly widespread. Two mutations, V1016G and F1534C, have functional effects on the sodium channel [13] and a third, S989P, may enhance 
resistance if found in conjunction with the V1016G mutation [14]. In southeast Asia, the frequencies of resistance alleles of these three mutations in Ae. aegypti vary between sites, but tend to be common. In Indonesia, the V1016G mutation and the associated S989P mutation are more common than F1534C [14,15]. The V1016G mutation is widely distributed and has been detected in Ae. aegypti from Malaysia, Indonesia, Thailand, Singapore and China [16]. F1534C is common in Ae. aegypti from Thailand [17], Vietnam [18], and further afield in the Caribbean [19] and Brazil [20]. V1016G is often associated with S989P and, in parts of southeast Asia and throughout the Indo-Pacific region, the 1016G resistant homozygote appears to be tightly linked to the F1534 susceptible homozygote [14,21,22]. The co-occurrence of homozygous mutations of 1016G/1534C/989P within an individual is rare, but has been detected at low frequency in Ae. aegypti populations in Myanmar [23], Indonesia [15] and Saudi Arabia [24].

The current study aimed to screen samples of Ae. aegypti and Ae. albopictus from Kuala Lumpur for sodium channel mutations that can be used as a convenient marker for one mechanism of pyrethroid insecticide resistance in mosquitoes in this location where a Wolbachia mosquito population replacement strategy is in progress [25]. In detail, we aimed to:

1. Monitor insecticide resistance in Aedes aegypti in the vicinity of Wolbachia program release sites in Shah Alam, Selangor (30 km from Kuala Lumpur) and look for changes in the genetic constitution of resistance at those sites.

2. Characterize the genotypes at three loci in the sodium channel for the V1016G, F1534C and S989P mutations and assess the association between the mutations and pyrethroid resistance in Ae. aegypti in Shah Alam.

3. Assess the utility of backcrossing laboratory colony Wolbachia mosquitoes to the field population to maintain consistency in sodium channel mutations and enhance survival of the released mosquitoes.

4. Sequence the region of the sodium channel gene around codon 1534 in Ae. albopictus (a second vector of dengue which may be the target of future Wolbachia release programs in Malaysia) from AU2, Keramat, Selangor (Kuala Lumpur boundary) to look for resistance mutations which have been identified in some populations, though not yet from Malaysia.

The momentum for this work stems from a Wolbachia release program being undertaken in Kuala Lumpur where uninfected $A$ e. aegypti populations are currently being replaced by $w \mathrm{AlbB}$ Wolbachia-infected mosquitoes [25], which can prevent the transmission of dengue and other arboviruses [26]. A key aspect to the success of Wolbachia releases is to ensure that the genetic background of the release strain is similar to that of the target population $[27,28]$, which contributes to production and release of competitive mosquitoes. While Wolbachia does not directly affect resistance in Ae. aegypti [29], matching insecticide resistance to background levels is a key component of the genetic background given that releases can fail if there is inadequate insecticide resistance, as happened in wMel Wolbachia releases in Rio de Janeiro [30,31]. Monitoring of resistance in release stock can be greatly facilitated by using molecular markers and we therefore had the subsidiary aim of maintaining the relevant sodium channel mutations in Wolbachia-infected mosquito strains being used for release in Malaysia to ensure that these strains are not disadvantaged relative to resident populations under current patterns of pyrethroid insecticide use.

\section{Materials and Methods}

\subsection{Sampling and the Wolbachia Release Program}

The sampling sites were PKNS, Shah Alam (area: $0.60 \mathrm{~km}^{2}$ ), a mix of 51 blocks of 5-storey low rise flats and AU2, Keramat, (area: $0.71 \mathrm{~km}^{2}$ ), a housing estate with landed property of terrace houses (Figure 1). 
A



B

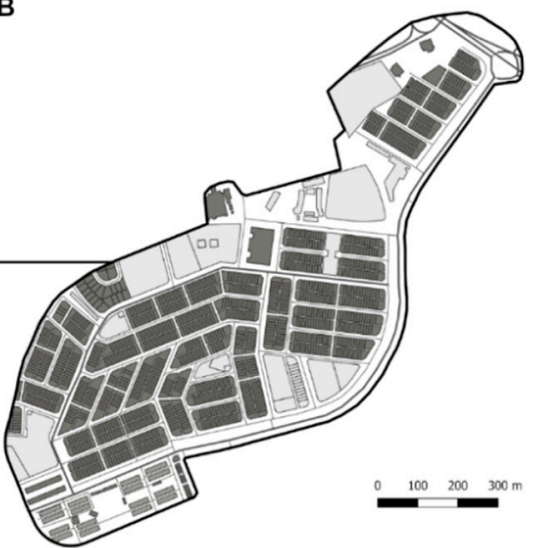

Figure 1. Map of collection sites of Aedes aegypti and Ae. albopictus, (A) PKNS, Shah Alam; (B) AU2, Keramat.

To collect Aedes spp., ovitrapping was conducted following the guidelines of the Ministry of Health on ovitrap deployment [32]. Black plastic containers of $300 \mathrm{~mL}$ volume were used as the ovitrap. Hardboard measuring $10 \mathrm{~cm} \times 2.5 \mathrm{~cm} \times 0.3 \mathrm{~cm}$ was used as an oviposition paddle and was placed in the ovitrap container to allow mosquitoes to lay eggs on its surface. Clean tap water was added to a level of $5.5 \mathrm{~cm}$ [33].

The surveillance at PKNS, Shah Alam was conducted on 14 November 2016, prior to the initiation of Wolbachia releases, and post-release on 11 February 2019. A total of 100 ovitraps were placed in covered areas on each occasion. Ovitraps were placed at the staircase and at the corridor of the flats in Shah Alam. In AU2, Keramat, ovitrapping was conducted on 16 May 2017. A total of 130 ovitraps were placed outdoors. After five days of exposure, the ovitraps were recovered and brought back to the Medical Entomology Unit laboratory, at the Institute for Medical Research (IMR) for species identification. Samples of Ae. aegypti from Shah Alam and Ae. albopictus from AU2 Keramat were reared for insecticide resistance testing and genotyping.

\subsection{Colony Samples}

Adult male and female Ae. aegypti were sampled over time from the laboratory colony used for the Wolbachia release program, which was maintained at several thousand individuals across generations. Mosquitoes from the colony are almost all infected with the Wolbachia strain wAlbB [25]. Generations sampled were F3, F8, F9, F13 and F20 reared since the third backcross of Wolbachia-infected females to field males (see Nazni et al. [25]). These stocks were designated B3F3 to B3F20, while the colony before backcrossing was designated as B0. A fourth and fifth backcross was conducted to produce stocks with the B5 designation and these were sampled directly after backcrossing (B5F0) and three generations later (B5F3). Mosquitoes from the IMR colony were preserved in absolute ethanol prior to DNA extraction and molecular screening for sodium channel mutations.

\subsection{Colony Rearing Protocols}

The $w$ AlbB infected mosquitoes were maintained at room temperature of $\sim 25{ }^{\circ} \mathrm{C}$ with $75 \pm 10 \%$ relative humidity and a photoperiod of 12:12 h (light/dark). Approximately 500 larvae were reared in a tray filled with $2000 \mathrm{~mL}$ of seasoned water (tap water stored overnight to dechlorinate). The immature stages of mosquitoes were supplied with liver powder (Difco ${ }^{\mathrm{TM}}$, Becton, Dickinson and Company, Franklin Lakes, NJ, USA) following the regime described by Nazni et al. [25] and adults were supplied with $10 \%$ sucrose solution incorporated with liquid B-complex. Adults were blood fed on white mice and allowed to lay eggs (Malaysian National Institute of Health approval number NMRR-16-297-28898). F1 females were then crossed to males of the field strain. An ovitrap container lined with damp filter 
paper (diameter, $12 \mathrm{~cm}$ ) was set as an oviposition site in each cage. After three days, the filter papers containing eggs were collected and allowed to air dry prior to use.

\subsection{Backcrossing}

For backcrossing, individual wAlbB infected females were sorted into glass tubes at the pupal stage. A total of 100 females emerged (aged 3-5 days) and were placed into a $24 \times 24 \times 24 \mathrm{~cm}$ sized cage. Wild males (aged 3-5 days) were put into the same cage at an equal ratio. A 10\% sucrose solution with a vitamin-B complex was supplied as an energy source. The mosquitoes were left for mating in the cage for 2-3 days prior to blood feeding. An ovitrap container lined with damp filter paper (diameter, $12 \mathrm{~cm}$ ) was set as an oviposition site in each cage. After three days, the filter papers containing eggs were collected and allowed to air dry prior to use.

\subsection{Adult Bioassays}

The adult mosquito bioassay was performed according to the standard WHO susceptibility or resistance test protocol [34,35]. A total of 15-20 female adults aged 3-5 days post-eclosion were used in the bioassays. Three test replicates and two controls were used. The adults were exposed to impregnated paper with diagnostic dosage, permethrin $0.25 \%$ or deltamethrin $0.03 \%$. Insecticide impregnated papers were purchased from the Vector Control Research Unit, Universiti Sains Malaysia, Penang.

In the tests, Ae. aegypti or Ae. albopictus were introduced into each tube. Exposure tubes with permethrin or deltamethrin impregnated papers were stood vertically throughout the test. Knockdown was scored every minute for $5 \mathrm{~min}$, followed by every $5 \mathrm{~min}$ for a $1 \mathrm{~h}$ exposure period. After exposure, the mosquitoes were transferred into paper cups and were supplied with cotton soaked in $10 \%$ sugar solution. Mortality was recorded after $24 \mathrm{~h}$. Classification of resistance status was made based on pre-determined WHO guidelines in which mortality of $<90 \%$ is characterized as resistant.

\subsection{Assays for Sodium Channel Mutations Associated with Pyrethroid Resistance}

\subsubsection{Aedes aegypti}

Three sodium channel (Vssc) mutation sites were screened in male and female Ae. aegypti on five occasions from the laboratory colony used for release (Backcross 3 F3, F8, F9 and F13 and Backcross 5 F0) and on males and females from field samples collected in November 2016 and September 2019 from PKNS, Shah Alam (see above). Dead and surviving female mosquitoes from the WHO bioassays (B3F9, B5F0 and field samples for permethrin; B3F20, B5F3 and field samples for deltamethrin) were also screened. The codon positions relating to the mutation sites in this study are labelled as S989P, V1016G and F1534C according to the sequence of the most abundant splice variant of the house fly, Musca domestica, VsSC (GenBank accession nos. AAB47604 and AAB47605) [36]. These mutation sites are equivalent to those in other studies that are labelled as S996P, V1023G and F1565C based on the VsSC homologue in Ae. aegypti, the AaNav protein (GenBank accession no. EU399181) [13].

Molecular screening of each mutation site in Ae. aegypti was achieved using Custom TaqMan ${ }^{\circledR}$ Single Nucleotide Polymorphism (SNP) Genotyping Assays (Life Technologies Corporation, Carlsbad, CA, USA) developed using sequence data from Wuliandari et al. [14]. Primers, probe sequences and amplicon lengths for each mutation site $(989,1016,1534)$ are described by Endersby-Harshman et al. [22].

A LightCycler ${ }^{\circledR}$ II 480 (Roche, Basel, Switzerland) real time PCR machine with a 384-well format was used to run three replicates of each SNP assay. The PCR Master Mix consisted of 40x TaqMan ${ }^{\circledR}$ assay $(0.174 \mu \mathrm{L}), 2 \times$ KAPA Fast PCR Probe Force qPCR Master Mix (KAPABIOSYSTEMS, Cape Town, South Africa) $(3.5 \mu \mathrm{L}), \mathrm{ddH}_{2} \mathrm{O}$ and genomic DNA diluted 1:5 in water $(2 \mu \mathrm{L})$. Cycling conditions for the PCR were pre-incubation of $3 \mathrm{~min}$ at $98^{\circ} \mathrm{C}$ (ramp rate $4.8^{\circ} \mathrm{C} / \mathrm{s}$ ) followed by 40 cycles of amplification at $95{ }^{\circ} \mathrm{C}$ for $10 \mathrm{~s}\left(2.5^{\circ} \mathrm{C} / \mathrm{s}\right.$ ramp rate) and $60{ }^{\circ} \mathrm{C}$ for $20 \mathrm{~s}\left(2.5^{\circ} \mathrm{C} / \mathrm{s}\right.$ ramp rate $)$ (Acquisition mode: single) 
with a final cooling step of $37^{\circ} \mathrm{C}$ for $1 \mathrm{~min}\left(2.5^{\circ} \mathrm{C} / \mathrm{s}\right.$ ramp rate). The Roche LightCycler ${ }^{\circledR} 480$ Software Version 1.5.1.62 was used to conduct endpoint genotyping.

\subsubsection{Aedes albopictus}

The Vssc codon 1534 in S6, Domain III of Ae. albopictus was screened for mutations using a forward primer designed to bind in the exon which contains codon 1534: (Alb171F5'CCGATTCGCGAGACCAACAT3') and the reverse primer of Kasai et al. [37] (aegSCR8). An amount of $2 \mu \mathrm{L}$ of genomic DNA, extracted using Chelex ${ }^{\circledR}$ resin (Bio-Rad Laboratories, Hercules CA, USA) and diluted 1:10, was amplified in a $25 \mu \mathrm{L}$ PCR mix that included final concentrations of ThermoPol buffer Mg-free (1x) (New England Biolabs, Ipswich, MA, USA), dNTPs (200 $\mu \mathrm{M}$ each) (Bioline, London, $\mathrm{UK}), \mathrm{MgCl}_{2}(1.5 \mathrm{mM}), 0.5 \mu \mathrm{M}$ each of forward and reverse primers, 0.625 units of Immolase ${ }^{\mathrm{TM}} \mathrm{Taq}$ polymerase (Bioline, London, UK), and PCR-grade $\mathrm{H}_{2} \mathrm{O}$. Thermocycling conditions for the PCR were initial denaturation at $95^{\circ} \mathrm{C}$ for $10 \mathrm{~min}, 35$ cycles at $95^{\circ} \mathrm{C}$ for $30 \mathrm{~s}$, annealment at $52{ }^{\circ} \mathrm{C}$ for $45 \mathrm{~s}$ and extension at $72{ }^{\circ} \mathrm{C}$ for $45 \mathrm{~s}$, followed by a final extension of $5 \mathrm{~min}$ at $72{ }^{\circ} \mathrm{C}$ before cooling to $10{ }^{\circ} \mathrm{C}$. PCR amplicons (220 bp) were sent to 1st BASE DNA Sequencing (Selangor, Malaysia) for Sanger sequencing on a 3730xl DNA analyzer. Sequences (up to $180 \mathrm{bp}$ ) were aligned and analyzed using the program Geneious ${ }^{\circledR}$ 11.1.4 (Biomatters, Ltd., Auckland, New Zealand).

\subsection{Analyses}

The odds ratio is a measure of association or relationship between two categorical variables. In this case, we use the odds ratio to assess whether there was an association between the categorical variables genotype and phenotype. The genotype for some of the tests is the VsSC mutation profile and the phenotype is whether the mosquito died or survived the diagnostic dose of permethrin or deltamethrin. For other tests, the phenotype is either male or female and, in some cases the odds ratio was determined as the odds of surviving as one genotype rather than another. Chi-squared tests of independence were conducted to explore relationships between categorical variables using IBM $^{\circledR}$ SPSS ${ }^{\circledR}$ Statistics Version 24 and with probabilities determined by the Monte Carlo procedure.

\section{Results}

\subsection{Adult Bioassays}

Adult female Ae. aegypti from the field strain collected at Shah Alam without Wolbachia and tested at the F1-F3 generations were highly resistant to $0.25 \%$ permethrin delivered on impregnated filter papers in WHO diagnostic dose bioassays as reflected by a low mortality, although the mean mortality did tend to vary across generations (Figure 2a). The proportion surviving the treatment was higher than the proportion that died on all occasions, although there was some heterogeneity in numbers across multiple bioassays done on the F2 generation (contingency test, $\chi^{2}=16.7 \mathrm{df}=3, p<0.01$ ). Over multiple generations in the laboratory (F1-F6), the Shah Alam field colony did vary in mortality (3.33 to $25.93 \%$ ), but mortality did not change consistently over time. On the WHO bioassay scale, mortality of $<90 \%$ is characterized as resistant. Permethrin bioassays of the Wolbachia wAlbB release strain of Ae. aegypti over multiple generations following backcrossing (B3F1-B3F11) also showed a high level of resistance, with mortality ranging from 5 to $29.63 \%$, and again there was no consistent trend over time (Figure $2 b$ ).

Bioassays on the field strain of Ae. albopictus indicated resistance to permethrin based on the WHO categorization, but not to the same extent as Ae. aegypti (52\% mortality (45.5-58.7, 95\% confidence intervals) at $0.25 \%$ permethrin compared with $9.5 \%$ mortality of Ae. aegypti). However, no significant difference between proportions of these species that were dead or survived was detected $\left(\chi^{2}=2.09\right.$, $\mathrm{df}=1, p>0.05)$.

Resistance to deltamethrin $(0.03 \%)$ was detected by bioassays of Ae. aegypti. The laboratory colony at B3F20 showed $84.8 \%$ mortality, which dropped somewhat to $63.81 \%$ after further backcrossing 
(B5F3). A recent field colony, established from mosquitoes collected from Shah Alam where releases were terminated about 1.5 years ago and then reared for four generations in the lab (i.e., at the F4 generation), showed a similar mortality of $71.8 \%$.



(a)

Figure 2. Cont.

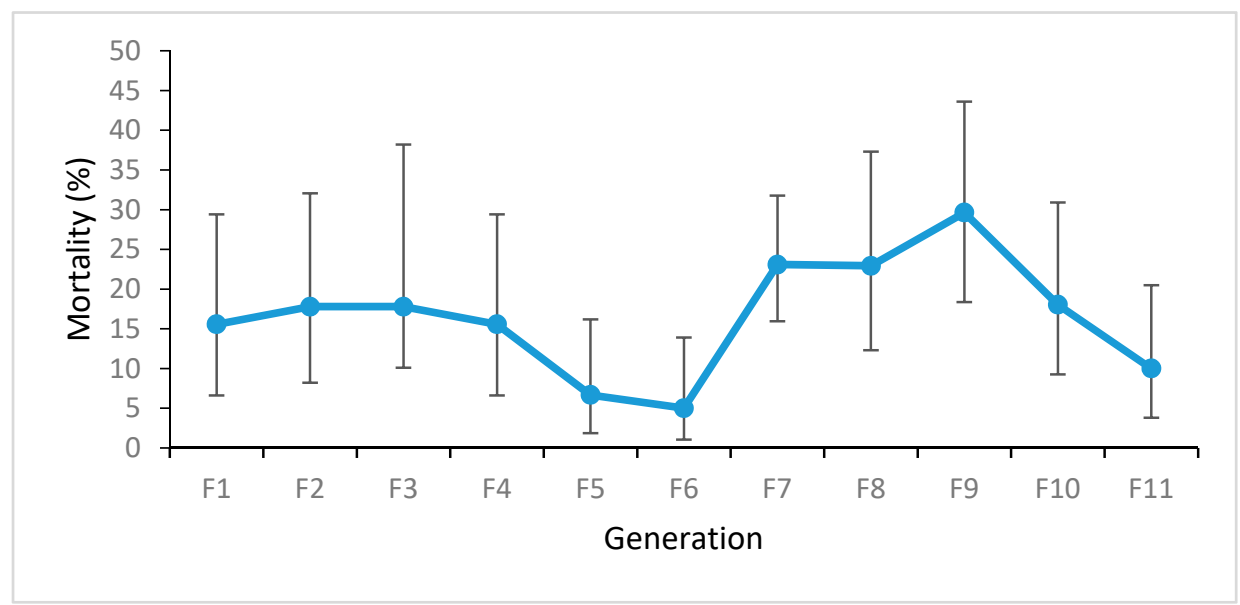

(b)

Figure 2. Mortality (\%) of female Ae. aegypti adults from (a) the field strain from Shah Alam during the F1 to F6 generations in the laboratory, and (b) the wAlbB strain from B3F1 to B3F11 generations. Mortality was tested with WHO bioassay impregnated papers (permethrin $0.25 \%$ ). Error bars represent 95\% binomial confidence intervals.

\subsection{Assays for Sodium Channel Mutations Associated with Pyrethroid Resistance}

Genotypes were compared among surviving and dead female Ae. aegypti from Shah Alam after bioassays with permethrin. These mosquitoes were tested prior to release of Wolbachia-infected mosquitoes, and showed the presence of three Vssc genotypes (GG/TT/CC, TT/GG/TT, TG/TG/TC-order 1016/1534/989) (Table 1), each of which would be expected to confer some level of resistance to pyrethroids. The odds of having the mutant genotype (GG or CC) and surviving were not different 
from survival without these mutations when considering single mutation sites at a time (1016 or 989: odds of surviving if mutant homozygote $=0.12(0.01-1.03,95 \%$ c.i. $)$ and $1534 \mathrm{OR}=0.89(0.35-2.29$, $95 \%$ c.i.), NS $=$ not statistically significant at $\alpha=0.05-$ c.i. values encompass 1.0$)$. However, if genotypes for the three mutation sites are considered together, the odds of surviving $0.25 \%$ permethrin were significantly higher for a triple heterozygote than for a wildtype genotype (Table 2). TT/GG/TT individuals also had greater odds of survival than wildtype mosquitoes $(\alpha=0.05-$ c.i. values do not encompass 1.0) (Table 2).

Table 1. VsSc genotypes of survivors and dead female Aedes aegypti from the field and the laboratory release colony from WHO bioassays with permethrin (0.25\%) (order of mutation sites 1016/1534/989) (Field = Shah Alam 2016 pre-release and Shah Alam 2019 after release; the laboratory release colony was tested at B3F9 and B5F0).

\begin{tabular}{ccccccccc}
\hline & \multicolumn{7}{c}{ Genotype Frequency (\%) (n) } \\
\cline { 3 - 9 } & & GG/TT/CC TT/GG/TT & TG/TG/TC & TG/TT/TC & TT/TG/TT & TT/TT/TT & TG/TG/TT \\
\hline Gen & A & B & C & D & E & F & H \\
\hline Survivors & Field 2016 & $2.5(1)$ & $30.0(12)$ & $67.5(27)$ & $0.0(0)$ & $0.0(0)$ & $0.0(0)$ & $0.0(0)$ \\
Dead & Field 2016 & $17.5(7)$ & $32.5(13)$ & $50.0(20)$ & $0.0(0)$ & $0.0(0)$ & $0.0(0)$ & $0.0(0)$ \\
Survivors & Field 2019 & $15.0(6)$ & $35.0(14)$ & $45.0(18)$ & $2.5(1)$ & $2.5(1)$ & $0.0(0)$ & $0.0(0)$ \\
Dead & Field 2019 & $12.5(5)$ & $37.5(15)$ & $35.0(14)$ & $0.0(0)$ & $12.5(5)$ & $2.5(1)$ & $0.0(0)$ \\
\hline Survivors & Release B3F9 & $2.5(1)$ & $15.0(6)$ & $15.0(6)$ & $5.0(2)$ & $52.5(21)$ & $10.0(4)$ & $0.0(0)$ \\
Dead & Release B3F9 & $2.5(1)$ & $10.0(4)$ & $10.0(4)$ & $10.0(4)$ & $35.0(14)$ & $32.5(13)$ & $0.0(0)$ \\
Survivors & Release B5F0 & $2.5(1)$ & $42.5(17)$ & $27.5(11)$ & $5.0(2)$ & $22.5(9)$ & $0.0(0)$ & $0.0(0)$ \\
Dead & Release B5F0 & $5.0(2)$ & $35.0(14)$ & $20.0(8)$ & $0.0(0)$ & $35.0(14)$ & $0.0(0)$ & $5.0(2)$ \\
\hline
\end{tabular}

Table 2. Odds ratio comparisons of genotypes of Aedes aegypti $(\mathrm{n}=322)$ from field and laboratory colonies (permethrin bioassay results combined). Odds ratio (OR) of surviving $0.25 \%$ permethrin with a specific genotype (order of mutation sites 1016/1534/989) ( $n=$ number of each genotype) are presented.

\begin{tabular}{|c|c|c|c|c|c|c|}
\hline \multicolumn{2}{|c|}{ Odds of Surviving } & \multirow{2}{*}{$\mathbf{n}$} & \multirow{2}{*}{ OR } & \multicolumn{2}{|c|}{$95 \%$ Confidence Interval } & \multirow{2}{*}{$\begin{array}{c}\text { OR Significant } \\
\quad \alpha=0.05\end{array}$} \\
\hline If: & Rather Than: & & & Lower & Upper & \\
\hline TG/TG/TC & $\mathrm{TT} / \mathrm{TT} / \mathrm{TT}$ & 108,18 & 4.72 & 1.46 & 15.27 & * \\
\hline TG/TT/TC & $\mathrm{TT} / \mathrm{TT} / \mathrm{TT}$ & 9,18 & 4.38 & 0.78 & 24.47 & \\
\hline TT/GG/TT & $\mathrm{TT} / \mathrm{TT} / \mathrm{TT}$ & 95,18 & 3.73 & 1.14 & 12.16 & * \\
\hline TT/TG/TT & TT/TT/TT & 64,18 & 3.29 & 0.98 & 11.08 & \\
\hline GG/TT/CC & TT/TT/TT & 24,18 & 2.10 & 0.53 & 8.39 & \\
\hline TG/TG/TC & GG/TT/CC & 108,24 & 2.25 & 0.90 & 5.58 & \\
\hline TG/TT/TC & GG/TT/CC & 9,24 & 2.08 & 0.44 & 9.84 & \\
\hline TT/GG/TT & GG/TT/CC & 95,24 & 1.78 & 0.71 & 4.45 & \\
\hline TT/TG/TT & GG/TT/CC & 64,24 & 1.57 & 0.60 & 12.09 & \\
\hline TG/TG/TC & TT/GG/TT & 108,95 & 1.27 & 0.73 & 2.20 & \\
\hline TT/GG/TT & TT/TG/TT & 95,64 & 1.13 & 0.60 & 2.14 & \\
\hline TG/TG/TC & TG/TT/TC & 108,9 & 1.08 & 0.27 & 4.24 & \\
\hline
\end{tabular}

The odds of surviving $0.03 \%$ deltamethrin were greatly enhanced for mosquitoes with the mutant genotypes TT/GG/TT or TG/TG/TC compared with wildtype or genotypes TG/TT/TC or TT/TG/TT (Table 3b).

Odds ratios could not be calculated for mosquitoes with genotype GG/TT/CC that were found in relatively low numbers in the sample, because no dead mosquitoes had this genotype (Figure 3).

In a comparison of VsSc genotypes found in the field (Shah Alam 2016) and colony (B3F3 IMR) samples of Ae. aegypti (Table 4), four Vssc genotypes were observed in both the field and colony samples (A = GG/TT/CC, B = TT/GG/TT, C = TG/TG/TC, E = TT/TG/TT) (order of mutation sites 1016/1534/989). An additional genotype ( $\mathrm{D}=\mathrm{TG} / \mathrm{TT} / \mathrm{TC}$ ) was found in mosquitoes from the colony. 
These five genotypes can be formed from three putative haplotypes (Figure 4). The frequency of the three most common genotypes in the field mosquitoes $(A, B, C)$ is similar to that in the release colony $\left(\chi^{2}=0.124, \mathrm{df}=2, p=0.940\right)$.

Table 3. (a) Vssc genotypes of survivors and dead female Aedes aegypti from the field and the laboratory release colony from WHO bioassays with deltamethrin (0.03\%) (order of mutation sites 1016/1534/989) (Field = Shah Alam F4, laboratory colony was tested at B3F20 and B5F3). (b) Odds ratios (OR) of surviving deltamethrin (type II pyrethroid) when a particular genotype is compared with another are also given.

\begin{tabular}{|c|c|c|c|c|c|c|c|c|}
\hline \multirow{2}{*}{ (a) } & & \multicolumn{7}{|c|}{ Genotype Frequency (\%) (n) } \\
\hline & & GG/TT/CC & \multirow{2}{*}{$\frac{\text { TT/GG/TT }}{\text { B }}$} & \multirow{2}{*}{$\frac{\text { TG/TG/TC }}{\text { C }}$} & \multirow{2}{*}{$\frac{\text { TG/TT/TC }}{\text { D }}$} & \multirow{2}{*}{$\begin{array}{c}\text { TT/TG/TT } \\
\text { E }\end{array}$} & \multirow{2}{*}{$\begin{array}{c}\mathrm{TT} / \mathrm{TT} / \mathrm{TT} \\
\mathrm{F}\end{array}$} & \multirow{2}{*}{$\frac{\mathrm{GG} / \mathrm{TT} / \mathrm{TC}}{\mathrm{G}}$} \\
\hline & Generation & A & & & & & & \\
\hline Survivors & Field F4 & $2.5(1)$ & $12.5(5)$ & $37.5(15)$ & $15.0(6)$ & $25.0(10)$ & $7.5(3)$ & $0.0(0)$ \\
\hline Dead & & $0.0(0)$ & $5.0(2)$ & $5.0(2)$ & $30.0(12)$ & $35.0(14)$ & $25.0(10)$ & $0.0(0)$ \\
\hline Survivors & $\begin{array}{c}\text { Release } \\
\text { B3F20 }\end{array}$ & $10.0(4)$ & $15.0(6)$ & $17.5(7)$ & $10.0(4)$ & $27.5(11)$ & $20.0(8)$ & $0.0(0)$ \\
\hline Dead & & $0.0(0)$ & $2.5(1)$ & $0.0(0)$ & $12.5(5)$ & $37.5(15)$ & $47.5(19)$ & $0.0(0)$ \\
\hline Survivors & $\begin{array}{c}\text { Release } \\
\text { B5F3 }\end{array}$ & $2.5(1)$ & $20.0(8)$ & $27.5(11)$ & $7.5(3)$ & $27.5(11)$ & $12.5(5)$ & $2.5(1)$ \\
\hline Dead & & $0.0(0)$ & $2.5(1)$ & $0.0(0)$ & $17.5(7)$ & $32.5(13)$ & $47.5(19)$ & $0.0(0)$ \\
\hline \multirow{12}{*}{ (b) } & \multicolumn{2}{|c|}{ Odds of Surviving } & \multirow[t]{2}{*}{ n } & \multirow[t]{2}{*}{ OR } & \multicolumn{2}{|c|}{$\begin{array}{l}\text { 95\% Confidence } \\
\text { Interval }\end{array}$} & \multirow{2}{*}{\multicolumn{2}{|c|}{$\begin{array}{l}\text { OR Significant } \\
\qquad \alpha=0.05\end{array}$}} \\
\hline & if: & rather than: & & & Lower & Upper & & \\
\hline & TT/GG/TT & TG/TG/TC & 23,35 & 0.29 & 0.05 & 1.72 & & \\
\hline & TT/GG/TT & TG/TT/TC & 23,37 & 8.77 & 2.46 & 31.29 & * & \\
\hline & $\mathrm{TT} / \mathrm{GG} / \mathrm{TT}$ & TT/TG/TT & 23,74 & 6.23 & 1.93 & 20.13 & * & \\
\hline & TT/GG/TT & $\mathrm{TT} / \mathrm{TT} / \mathrm{TT}$ & 23,64 & 14.25 & 4.22 & 48.16 & * & \\
\hline & TG/TG/TC & TG/TT/TC & 35,37 & 30.46 & 6.28 & 147.72 & * & \\
\hline & TG/TG/TC & TT/TG/TT & 35,74 & 21.66 & 4.83 & 97.02 & * & \\
\hline & $\mathrm{TG} / \mathrm{TG} / \mathrm{TC}$ & TT/TT/TT & 35,64 & 49.50 & 10.66 & 229.83 & * & \\
\hline & TG/TT/TC & TT/TG/TT & 37,74 & 0.71 & 0.31 & 1.61 & & \\
\hline & TG/TT/TC & $\mathrm{TT} / \mathrm{TT} / \mathrm{TT}$ & 37,64 & 1.63 & 0.67 & 3.92 & & \\
\hline & $\mathrm{TT} / \mathrm{TG} / / \mathrm{TT}$ & $\mathrm{TT} / \mathrm{TT} / \mathrm{TT}$ & 74,64 & 2.29 & 1.10 & 4.74 & * & \\
\hline
\end{tabular}

* Significant $(\alpha=0.05)$.

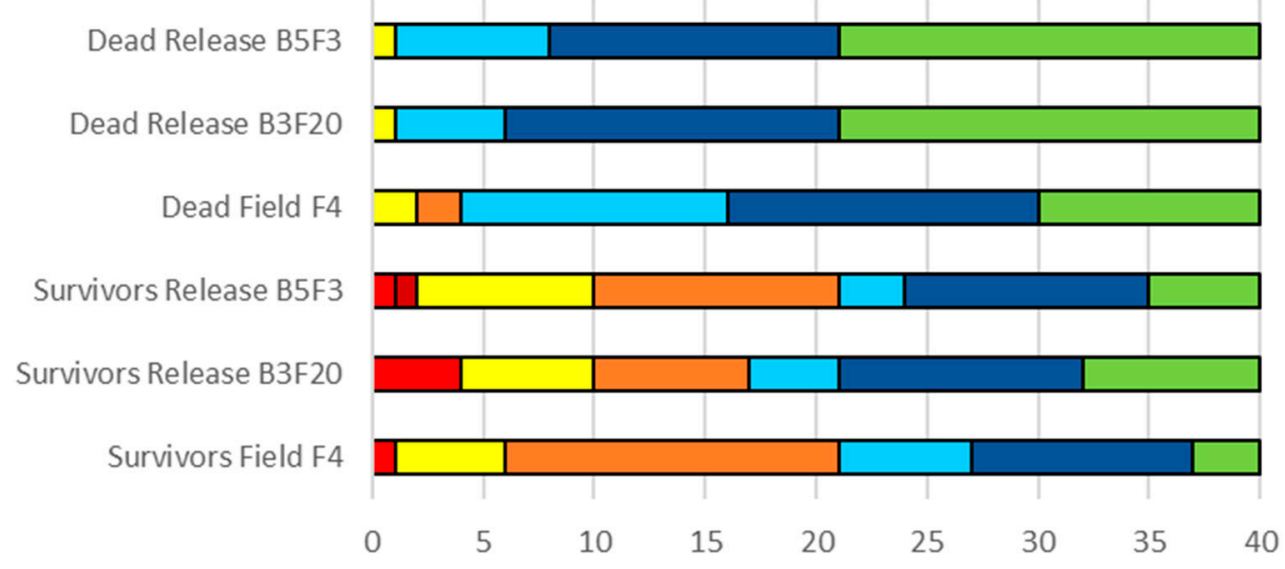

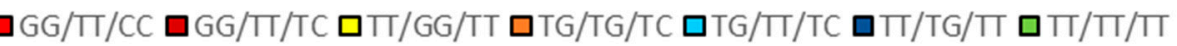

Figure 3. Proportion of each VSSC genotype in 40 samples of dead and surviving Aedes aegypti females from bioassays with deltamethrin (type II pyrethroid) (0.03\%). Mutations listed in order 1016/1534/989 (in all cases $\mathrm{T}=$ wildtype). 
Table 4. Comparison of VSSC genotypes found in field (Shah Alam, 2016) and colony (B3F3 IMR) samples of Aedes aegypti (order of mutation sites 1016/1534/989) at the time of initial Wolbachia releases.

\begin{tabular}{ccccccc}
\hline & \multicolumn{7}{c}{ Genotype Frequency (\%) (n) } \\
\cline { 2 - 7 } & GG/TT/CC & TT/GG/TT & TG/TG/TC & TG/TT/TC & TT/TG/TT & n \\
\hline $\mathbf{A}^{*}$ & $\mathbf{B}^{*}$ & $\mathbf{C}^{*}$ & $\mathbf{D}$ & $\mathbf{E}$ & \\
\hline Field Female & $15.00(6)$ & $20.00(8)$ & $60.00(24)$ & $0.0(0)$ & $5.00(2)$ & 40 \\
Field Male & $12.50(5)$ & $45.00(18)$ & $37.50(15)$ & $0.0(0)$ & $5.00(2)$ & 40 \\
Total & $13.75(11)$ & $32.50(26)$ & $48.75(39)$ & $0.0(0)$ & $5.00(4)$ & 80 \\
Colony & $15.00(6)$ & $5.00(2)$ & $47.50(19)$ & $7.50(3)$ & $25.00(10)$ & 40 \\
Female & $10.00(4)$ & $45.00(18)$ & $27.50(11)$ & $7.50(3)$ & $10.00(4)$ & 40 \\
Colony Male & $12.50(10)$ & $25.00(20)$ & $37.50(30)$ & $7.50(6)$ & $17.50(14)$ & 80 \\
Total & $12.00)$ &
\end{tabular}

* (Total field/total colony: $\chi^{2}=0.124, \mathrm{df}=2, p=0.940$; field female/field male: $\chi^{2}=6.014, \mathrm{df}=2, p=0.049$; colony female/colony male: $\left.\chi^{2}=14.882, \mathrm{df}=2, p=0.0006\right)$.



Figure 4. Putative Vssc haplotypes and observed genotypes in field (Shah Alam) (A-F) and IMR colony samples (A-G) of Aedes aegypti.

In both the Shah Alam field sample and the colony (B3F3), there were more female than male heterozygotes (TG/TG/TC) (Table 4).

Overall differences in genotype distribution between the sexes in the field were only marginally significant $\left(\chi^{2}=6.014, \mathrm{df}=2, p=0.049\right)$ for the common genotypes A, B and C. In the B3F3 colony, however, the genotype distribution (Figure $4 \mathrm{~A}-\mathrm{C}$, ) differed significantly between the sexes $\left(\chi^{2}=14.882\right.$, $\mathrm{df}=2, p=0.001)$. The odds of having genotype TT/GG/TT and being male were significantly different from expectations $(p<0.05)$ in both the field and the colony, but the odds ratio in the colony was higher (odds of having mutations GG at 1534 and being male: field (Shah Alam), OR = 3.27 (1.21-8.84, 95\% c.i.); colony B3F3, OR $=15.55$ (3.29-73.42, 95\% c.i.)). These odds ratios compared proportions of male TT/GG/TT out of males of other genotypes with the proportion of female TT/GG/TT out of females of other genotypes.

A test for the Hardy-Weinberg equilibrium (HWE) of the genotypes at the three Vssc mutation sites in Aedes aegypti from the field (Shah Alam), the laboratory (B3F3 IMR) and the bioassayed individuals (dead and alive) showed that the genotypes in the bioassay samples were not in HWE but the other samples were in HWE (Table S1). This deviation may reflect the fact that equal numbers of dead and surviving mosquitoes were genotyped, which meant that mosquito sampling in the bioassays was biased.

Comparison of VsSC mutations over time in the IMR Wolbachia release colony of Ae. aegypti showed changes in genotype distribution over generations in the B3 backcross (Table 5), which were significant by a contingency test for both females $\left(\chi^{2}=36.20, \mathrm{df}=10, p<0.001\right)$ and males $\left(\chi^{2}=27.08\right.$, $\mathrm{df}=10, p=0.002$ ). 
Table 5. Comparison of VSSC mutations across four generations of the IMR colony of Aedes aegypti (B3F3, B3F8, B3F13, B5F0) (order of mutation sites: 1016/1534/989).

\begin{tabular}{|c|c|c|c|c|c|c|c|}
\hline \multirow[b]{2}{*}{ Genotype } & \multicolumn{7}{|c|}{ Genotype Frequency (\%) (n) } \\
\hline & $\begin{array}{c}\text { GG/TT/CC } \\
\text { A }\end{array}$ & $\begin{array}{c}\text { TT/GG/TT } \\
\text { B }\end{array}$ & $\begin{array}{c}\text { TG/TG/TC } \\
\text { C }\end{array}$ & $\begin{array}{c}\text { TG/TT/TC } \\
\text { D }\end{array}$ & $\begin{array}{c}\text { TT/TG/TT } \\
\text { E }\end{array}$ & $\begin{array}{c}\text { TT/TT/TT } \\
\text { F }\end{array}$ & n \\
\hline $\begin{array}{c}\text { B3F3 } \\
\text { Female }\end{array}$ & $15.0(6)$ & $5.0(2)$ & 47.5 (19) & $7.5(3)$ & $25.0(10)$ & $0.0(0)$ & 40 \\
\hline B3F3 Male & $10.0(4)$ & $45.0(18)$ & $27.5(11)$ & $7.5(3)$ & $10.0(4)$ & $0.0(0)$ & 40 \\
\hline $\begin{array}{c}\text { B3F8 } \\
\text { Female }\end{array}$ & $0(0)$ & $13.3(4)$ & $20.0(6)$ & $13.3(4)$ & $43.3(13)$ & $10.0(3)$ & 30 \\
\hline B3F8 Male & $7.1(2)$ & 46.4 (13) & $3.6(1)$ & $7.1(2)$ & $21.4(6)$ & $14.3(4)$ & 28 \\
\hline $\begin{array}{c}\text { B3F13 } \\
\text { Female }\end{array}$ & $5.0(2)$ & $12.5(5)$ & $7.5(3)$ & $7.5(3)$ & $50.0(20)$ & 17.5 (7) & 40 \\
\hline $\begin{array}{l}\text { B3F13 } \\
\text { Male }\end{array}$ & $2.5(1)$ & $35.0(14)$ & $5.0(2)$ & $10.0(4)$ & $32.5(13)$ & $15.0(6)$ & 40 \\
\hline $\begin{array}{c}\text { B5F0 } \\
\text { Female }\end{array}$ & $7.5(3)$ & $25.0(10)$ & $37.5(15)$ & $7.5(3)$ & $22.5(9)$ & $0.0(0)$ & 40 \\
\hline B5F0 Male & $0.0(0)$ & $27.5(11)$ & 37.5 (15) & $22.5(9)$ & $12.5(5)$ & $0.0(0)$ & 40 \\
\hline
\end{tabular}

Wildtype individuals (TT/TT/TT) were not found in the B3F3 baseline population, but were common in both sexes in B3F8. TT/TG/TT was the most common female genotype in B3F8, whereas the triple heterozygote (TG/TG/TC) had been the most common in B3F3. TT/GG/TT remained the most common genotype in males between $\mathrm{B} 3 \mathrm{~F} 3$ and $\mathrm{B} 3 \mathrm{~F} 8$, but the prevalence of triple heterozygotes declined and TT/TG/TT increased as it did in females. After ten generations without backcrossing to field mosquitoes (B3F3 to B3F13), changes in the VSSC mutation profile became apparent (Figure 5). No wildtype mosquitoes were observed in the baseline population, but at B3F13, they comprised $16 \%$ of the samples screened $(n=80)$. The triple heterozygote decreased in frequency $(38 \%$ to $6 \%)$ and the 1016/989 mutation (genotype A), which did not occur in large numbers, also decreased by two thirds. The 1534 mutation (genotype B) was maintained in the laboratory colony, but the heterozygote (genotype E) increased (18\% to $41 \%)$. These results point to a decrease in the resistant genotypes and alleles.

A fourth and fifth backcross was undertaken, and in the B5F0 mosquitoes, the homozygous 1016 mutation was not found in the sample of males (Table 5). After further backcrossing, the sex difference in the frequency of the 1534 mutation was no longer evident, and the prevalence of the TT/TG/TT genotype decreased overall. Wildtype individuals (TT/TT/TT) were not observed in the backcrossed sample, while the triple heterozygote, TG/TG/TC, became more abundant. The backcrossing introduced two new genotypes to the laboratory colony, $\mathrm{H}=\mathrm{TG} / \mathrm{TG} / \mathrm{TT}$ (Table 1) and $\mathrm{G}=\mathrm{GG} / \mathrm{TT} / \mathrm{TC}$ (Table 3a), both of which include a fourth haplotype (H4-Figure 4) not identified in the field sampling, but clearly present in the wild males used for the cross. The additional backcross generations therefore reintroduced resistant alleles into the population.

A comparison of the distribution of Vssc genotypes (A, B, C) at the field site, Shah Alam, in 2016 before the Wolbachia release and in 2019 after release, showed that the distribution of these genotypes had remained the same $\left(\chi^{2}=3.91, \mathrm{df}=2, p=0.141\right)$ (Table 6). 


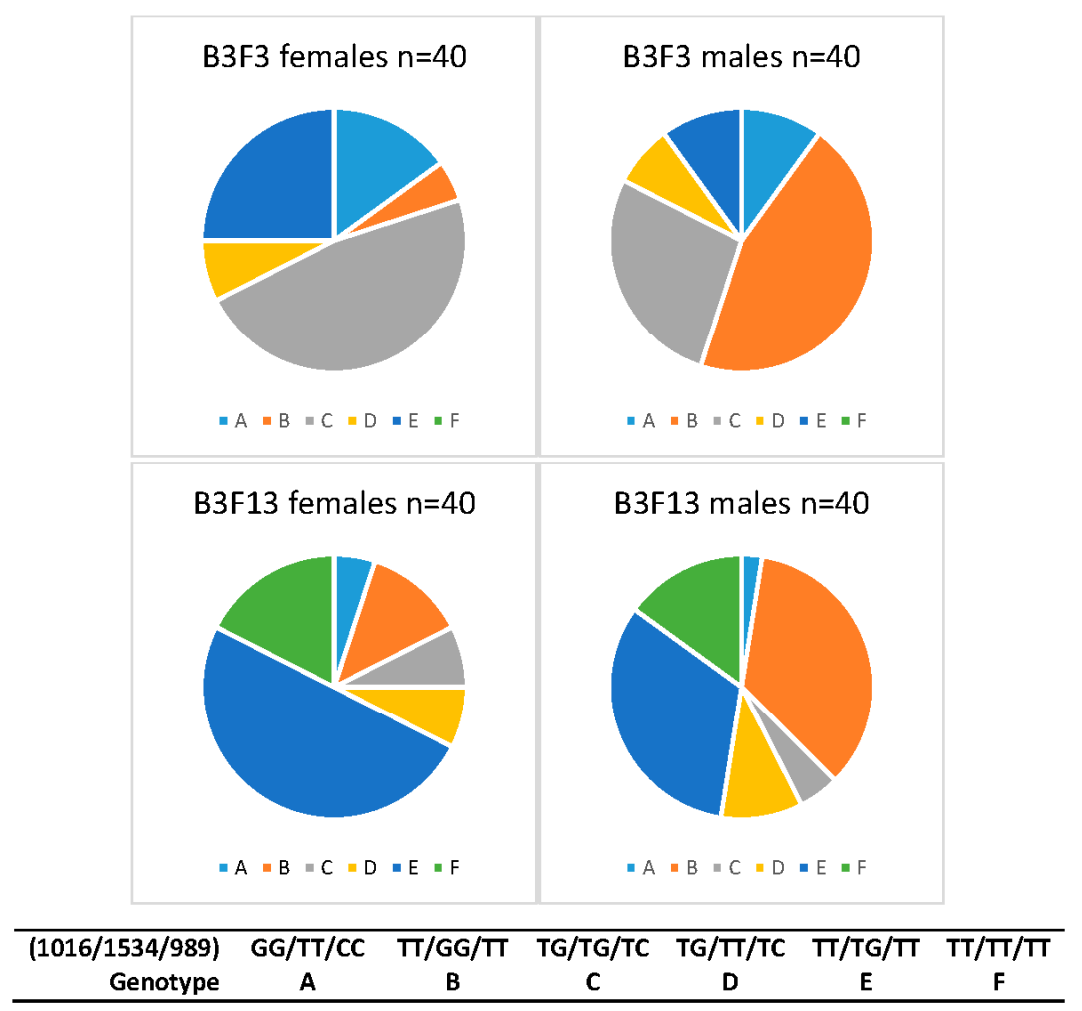

Figure 5. Changes in frequency of VSSC genotypes between B3F3 and B3 F13 (ten generations) of the IMR laboratory colony (wAlbB infected) and differences between female and male Aedes aegypti.

Table 6. Comparison of VsSc genotypes found in the field (PKNS Shah Alam) in 2016 (pre-release) and 2019 (post-release of Wolbachia) in Ae. aegypti.

\begin{tabular}{|c|c|c|c|c|c|c|c|c|}
\hline & & \multicolumn{7}{|c|}{ Genotype Frequency (\%) (n) } \\
\hline & & GG/TT/CC & $\mathrm{TT} / \mathrm{GG} / \mathrm{TT}$ & TG/TG/TC & TG/TT/TC & TT/TG/TT & $\mathrm{TT} / \mathrm{TT} / \mathrm{TT}$ & $\mathbf{n}$ \\
\hline & & A & B & $\mathrm{C}$ & D & $\mathrm{E}$ & F & \\
\hline $\begin{array}{l}\text { Field } \\
\text { Female }\end{array}$ & 2016 & $15.00(6)$ & $20.00(8)$ & $60.00(24)$ & $0.0(0)$ & $5.00(2)$ & $0.00(0)$ & 40 \\
\hline $\begin{array}{l}\text { Field } \\
\text { Male }\end{array}$ & & $12.50(5)$ & $45.00(18)$ & $37.50(15)$ & $0.0(0)$ & $5.00(2)$ & $0.00(0)$ & 40 \\
\hline Total & & $13.75(11)$ & $32.50(26)$ & $48.75(39)$ & $0.0(0)$ & $5.00(4)$ & $0.00(0)$ & 80 \\
\hline $\begin{array}{l}\text { Field } \\
\text { Female }\end{array}$ & 2019 & $15.00(6)$ & $32.50(13)$ & $35.00(14)$ & $2.50(1)$ & $12.50(5)$ & $2.50(1)$ & 40 \\
\hline $\begin{array}{l}\text { Field } \\
\text { Male }\end{array}$ & & $25.00(10)$ & $40.00(16)$ & $27.50(11)$ & $0.00(0)$ & $7.50(3)$ & $0.00(0)$ & 40 \\
\hline Total & & $20.00(16)$ & $36.25(29)$ & $31.25(25)$ & $1.25(1)$ & $10.00(8)$ & $1.25(1)$ & 80 \\
\hline
\end{tabular}

Note: Distribution of genotypes A, B, C in 2016 compared with $2019 ; \chi^{2}=3.91, \mathrm{df}=2, p=0.141$.

\subsection{Aedes albopictus}

The DNA sequences for 27 individuals of Aedes albopictus from AU2, Keramat, for a small section of the voltage-sensitive sodium channel gene (Vssc) from S6, domain III, were obtained (Supplementary Material S1). The wildtype state at codon 1534 was TTC (phenylalanine). A total of 23 individuals showed this genotype (GenBank Accession numbers: (MK992016, MK992018, MK992020-MK992029, MK992031-MK992033, MK992035-MK992042). Sample E09 (MK992030) showed a non-synonymous homozygous mutation at codon 1534 (TTG/TTG) that would code for leucine. Samples A10 (MK992017), 
B07 (MK992019), and F10 (MK992034) were heterozygotes at codon 1534 (TTC/TTG) (IUPAC code S for $\mathrm{C} / \mathrm{G}$ appears in the sequence).

Synonymous mutations (TTT and TTC) were found at codon 1528, some of which comprised heterozygotes (IUPAC code Y for C/T) (13 individuals). Sample E07 (MK992029) was heterozygous at position three in codon 1539 (IUPAC code $\mathrm{Y}$ for C/T), but it is a synonymous mutation. Samples A01 (MK992016), B05 (MK992018), B10 (MK992021), C6 (MK992024) and E4 (MK992028) were heterozygotes at codon 1544 position three (IUPAC code $\mathrm{Y}$ for $\mathrm{C} / \mathrm{T}$ ). The $\mathrm{T}$ is a synonymous mutation (isoleucine). The same five samples were also heterozygous at codon 1561, position 3 (IUPAC code K for G/T), another synonymous mutation (serine). Another heterozygote occurred at position three, codon 1547, in sample B08 (MK992020) (IUPAC code Y for C/T). The alternatives are again synonymous (isoleucine). Sample G09 (MK992038) was heterozygous at codon 1582, position three (IUPAC code R for A/G), but the mutation is synonymous (leucine).

\section{Discussion}

Pressure from pyrethroid insecticide applications can be intensive in regions where mosquitoes involved in transmission of dengue (Ae. aegypti and Ae. albopictus) are common, and selection under these conditions seems inevitable, with very few exceptions where pesticide applications have been restricted [38]. Alternative dengue control programs, which involve release of dengue-blocking Wolbachia-infected mosquitoes to replace natural populations, can reduce insecticide applications as mosquitoes reduce their virus-transmitting competence [26,39]. However, during the establishment phase of these programs, it is likely that conventional control options such as pesticide application will continue to be required, potentially jeopardizing survival of the laboratory-reared mosquitoes and threatening the success of Wolbachia release programs [31]. To mitigate this problem, Wolbachia mosquitoes are backcrossed to field individuals multiple times to ensure they have an equivalent genetic background [27] which, amongst other traits, will include resistance to the level of insecticide pressure experienced at the release sites. However, resistance can be lost from release populations, even after backcrossing, if there are costs associated with resistance alleles [30,31].

A Wolbachia mosquito release program for Ae. aegypti conducted around Kuala Lumpur in Malaysia from 2016 to the present day has been successful both in establishment of the Wolbachia mosquitoes and in reducing the number of dengue cases in release areas [25]. Throughout the establishment of the program, there was an opportunity to study various aspects of mosquito releases. Our study focused on the effects of continued pyrethroid applications around the release areas, the resistance genotypes and phenotypes of field and laboratory mosquitoes and benefits of regular backcrossing to field mosquitoes compared with long-term mosquito rearing as a closed population.

\subsection{Resistance in the Dengue Vector Mosquitoes in Kuala Lumpur}

We found phenotypic resistance to both type I and II pyrethroids in Ae. aegypti and resistance to at least type I pyrethroids in Ae. albopictus in the field. Resistance to both type I and II pyrethroids was also observed in release colony samples of Ae. aegypti. Using three VsSC mutations as a proxy for pyrethroid resistance in general was less successful with the type I pyrethroid, permethrin, than with type II (deltamethrin), as there was only a weak correspondence between the presence of some genotypes and survival after permethrin exposure. The triple heterozygote (V1016G/F1534C/S989P) had a small survival advantage over the wildtype genotype (V1016/F1534/S989) and TT/GG/TT individuals also had a somewhat greater odds of survival than wildtype mosquitoes. In contrast, the odds of surviving deltamethrin $(0.03 \%)$ exposure were greatly enhanced by presence of the Vssc mutations, particularly the triple heterozygote and the homozygous mutations (GG/TT/CC and TT/GG/TT) which is consistent with findings of electrophysiological studies of these mutations expressed in Xenopus oocytes [13].

In bioassays, Aedes aegypti showed a higher level of resistance to permethrin compared with Ae. albopictus. This result is consistent with other studies [40] and most likely reflects a lower level of exposure to pyrethroids experienced by Ae. albopictus, as its habitat extends beyond the dwellings of 
humans where most insecticide applications take place [4]. With increased urbanization and increased exposure to pyrethroids by Ae. albopictus, the lower resistance levels in this species are likely to change and, in fact, appear to be doing so within southern and eastern China at present [41,42].

A newly discovered mutation, F1534L, in Ae. albopictus from Kuala Lumpur may be an indication that selection for higher levels of target-site resistance is underway in Malaysia as well. The F1534L mutation found in the Ae. albopictus samples in this study is the first sodium channel mutation to be reported from this species in Malaysia, even though extensive surveying was conducted in 2010 [4]. It appears that the resistance mutation in Ae. albopictus has increased to a detectable frequency since the 2010 samples were taken by Ishak et al. [4]. Ishak et al. [12] demonstrated that metabolic resistance caused by upregulation of the cytochrome P450, CYP6P12, was the main cause of resistance in $k d r$-free Ae. albopictus, but six years later, monitoring for $k d r$ also may be justified given that we have now detected a VSSC mutation.

A F1534L mutation has been found in Ae. albopictus in Florida, USA (2011) [43], but involves a TTC to CTC transition in the codon rather than the TTC to TTG mutation that we have detected. An F1534L mutation (TTC to TTG) has also been detected in Ae. albopictus from Guangzhou, China (2014), Shenzhen, China (2014) and from Arco, Italy (2011) [44]. The F1534L mutation is also now present in Ae. albopictus in Beijing [42]. Yan et al. [45] have made a functional study of this mutation and found that it reduced the VSSC sensitivity to type I, but not type II pyrethroids. Even so, Li et al. [46] found a significant association between F1534L and resistance to deltamethrin in Ae. albopictus.

VSSC mutations were found more commonly at codon 1534 than at the 1016 and 989 codons in Ae. aegypti from the release area around Kuala Lumpur. This result is consistent with that of Ishak et al. [4] who found the same trend in mosquitoes across Malaysia. The results are very different from similar studies of Ae. aegypti from Kedah and Johor states (the northern and southern regions of Malaysia) [47] as well as Yogyakarta [14], in which mutations at 1016 and 989 were common, but 1534 was rare. Within southeast Asia and the Indo-Pacific, the F1534C mutation in Ae. aegypti predominates also in Taiwan, Vietnam, Sri Lanka and Fiji, whereas in Bali (Indonesia) and Vanuatu, V1016G with S989P are the only mutations found [22].

\subsection{Comparison between Vssc Genotypes in the Release Colony and the Field}

In general terms, the Wolbachia release colony and field Ae. aegypti samples are very similar with respect to frequency of V1016G and S989P mutations, but, as mentioned above, these mutations occur at lower frequencies than F1534C in the tested samples. The Wolbachia release colony and field mosquitoes are also similar with respect to overall F1534C mutation frequency, although fewer homozygotes and more heterozygotes are found in the colony. This could have some impact on resistance level. The effects of VSSC mutations on fitness parameters not related to insecticide resistance are not known for Ae. aegypti, but a VSSC mutation in Anopheles gambiae, L1014F, has been shown to confer a male mating advantage in heterozygous individuals compared with males homozygous for the mutation and wildtype males [48].

The Wolbachia release colony at B3F3 had one combined genotype not found in the field (TG/TT/TC genotype $\mathrm{D}$, which looks like it received a wildtype haplotype (H3) from one parent and the $\mathrm{H} 1$ haplotype from the other). We found no individuals in the colony or field samples which were wildtype at all three loci at generation B3F3, however, with haplotype H3 being present in the colony, it is likely that they may exist at low frequency. Genotype E, found in the field, must also have had one parent that was wildtype or had a wildtype haplotype. In later samples (Field 2019), we found homozygous wildtype individuals in the field, meaning that there will still be some susceptibility to pyrethroid insecticides in Ae. aegypti in Kuala Lumpur. Wildtype homozygotes were also found in the release colony from B3F8 onwards, suggesting that this closed colony was changing over time with a low-frequency genotype reaching detectable levels. 


\subsection{Changes in Genotype Frequencies in the Release Strain over Time: Implications for Field Survival}

We detected a tendency for male Ae. aegypti in the field, but particularly the laboratory colony, to have a higher frequency of the F1534C homozygous mutation than females. Such a difference cannot be attributed to sex-linkage, as the Ae. aegypti sex-determining region is located on chromosome 1 and the VSSC gene is on chromosome 3 [36]. If males and females were coming from different populations as happens at each backcross event, this might provide an explanation. Otherwise, there might be environmental conditions where males and females are selected differently; perhaps a lethal effect of this mutation in females gives a sex-specific fitness cost. The conditions of colony rearing could make this cost higher than in the field. Though an interesting phenomenon, it only appears to occur sporadically, making further investigations difficult.

Between B3F3 and B3F8 of the Ae. aegypti Wolbachia release colony, there was a tendency for more susceptible genotypes to increase in frequency, and this continued further at B3F13 and B3F20. Resistance to type I pyrethroids is likely to have been maintained in the colony to some extent by 1534 in males, while the 1016 component was being lost. Though the colony was still classified as resistant according to $\mathrm{WHO}$, mortality caused by $0.03 \%$ deltamethrin was high at $84.8 \%$ in the closed Ae. aegypti colony at B3F20 compared with mortality levels with $0.25 \%$ permethrin. Resistance to this type II pyrethroid was clearly regained after backcrossing, as mortality dropped to 63.8\% (B5F3) and became equivalent to that of the recent field collection (F4), which showed mortality of $71.8 \%$ to deltamethrin in the same bioassay. In a newly backcrossed population (B5F0), the 1016 mutation did not increase in frequency and was not found at all in the sample of males. Backcrossing removed the sex difference in frequency of the 1534 mutation. The prevalence of the TT/TG/TT genotype decreased overall and wildtype individuals TT/TT/TT were not observed in the backcrossed sample despite having occurred at a high frequency in B3F3. By B5F3, however, wildtype homozygotes were detectable again. The implications of long-term rearing without backcrossing on resistance status of a release colony are demonstrated. We note the previous evidence for loss of resistance in wel Wolbachia infected lines in Brazil, which was associated with a failure to establish this infection in the field [30,31].

Releases of Wolbachia mosquitoes in the study area occurred between May 2017 and November 2017 as weekly events for a block of 26 weeks followed by a four-month gap and a second block of weekly releases for 31 weeks [25] using early generations of backcrossed material. Two years after release, Wolbachia infection of the mosquito population at the sites remained at $>90 \%$ [25] and VSSC mutations had not changed in distribution from our original screening before releases in 2016, demonstrating the value of keeping equivalent resistance mechanisms in the release population by backcrossing to mosquitoes from the field. While we focused on VsSc mutations and target-site resistance as a proxy measurement for pyrethroid resistance overall, metabolic resistance mechanisms in Ae. aegypti populations in Malaysia also play a significant role [4,11,12]; thus, analysis of these mechanisms would be a useful addition to further understand backcrossing requirements for Wolbachia release programs in both $A e$. aegypti and Ae. albopictus. In such analyses, it would be useful to make comparisons and undertake crosses to the well-characterized susceptible "Rockefeller" strain, which would also allow for more direct comparisons with other bioassay studies where this strain is often characterized.

\section{Conclusions}

We have identified VSSC mutations associated with pyrethroid resistance in populations of Aedes aegypti from Kuala Lumpur, Malaysia. Mosquitoes in two genotype classes demonstrated a type I pyrethroid resistance advantage over wildtype mosquitoes when exposed to $0.25 \%$ permethrin. This resistance advantage was even more pronounced with a type II pyrethroid, deltamethrin (0.03\%). A VsSc mutation in Aedes albopictus (F1534L), found for the first time in mosquitoes from Malaysia, suggests that selection for pyrethroid resistance in this second dengue vector species may be in progress.

Three Vssc haplotypes predominate in Ae. aegypti in this region and all persist in colonies with regular backcrossing to mosquitoes from the field. Changes in VSSC genotype frequency in closed 
populations of Ae. aegypti maintained for multiple generations in laboratory culture suggest different fitness costs associated with the genotypes, some of which may be associated with the sex of the mosquito. Following population replacement of Ae. aegypti by Wolbachia mosquitoes for dengue control using backcrossed populations, we found that the VSSC mutations have persisted at pre-release levels. The results point to the importance of VSSC mutations in pyrethroid resistance in mosquito populations and the need for regular backcrossing with male mosquitoes from the field to maintain similarity of genetic background and population integrity during Wolbachia mosquito releases.

Supplementary Materials: The following are available online at http://www.mdpi.com/2075-4450/11/8/529/s1, Table S1: Test for Hardy-Weinberg equilibrium of genotypes at three Vssc mutation sites in Aedes aegypti from the field (Shah Alam), the laboratory (B3F3 IMR) and bioassayed female individuals (dead and alive); Supplementary Material S1: DNA sequences for 27 individuals of Aedes albopictus for a small section of the voltage-sensitive sodium channel gene (Vssc) from S6, domain III from Shah Alam.

Author Contributions: Conceptualization, N.M.E.-H., A.A.H.; methodology, N.M.E.-H., N.A.A., N.R.M.M., S.N.S.A., S.P.S.; formal analysis, N.M.E.-H., A.A.H., N.A.A., N.R.M.M., N.Z.A.M.Z., S.N.S.A.; investigation, N.A.A., N.R.M.M., N.Z.A.M.Z., S.N.S.A., M.K.R.G., M.A.A.K.; resources, N.A.A., N.R.M.M., N.Z.A.M.Z., S.N.S.A., M.K.R.G., M.A.A.K., Y.L.C., S.P.S.; data curation, N.M.E.-H.; writing-original draft preparation, N.M.E.-H., N.A.A., N.W.A., N.R.M.M., N.Z.A.M.Z., M.K.R.G., M.A.A.K., Y.L.C.; writing-review and editing, N.A.A., N.M.E.-H., A.A.H., S.N.S.A.; Y.L.C., S.P.S.; visualization, N.M.E.-H., Y.L.C., A.A.H.; project administration, N.W.A., N.A.A., A.A.H., S.P.S.; funding acquisition, S.P.S., N.W.A., A.A.H. All authors have read and agreed to the published version of the manuscript.

Funding: This research was funded by the Wellcome Trust Award 108508, the National Health and Medical Research Council (Program Grants 1037003, 1132412) and the Ministry of Health Malaysia.

Acknowledgments: The authors thank the Director General of Health, Malaysia, for permission to publish. We acknowledge the Wolbachia team members from the Medical Entomology Unit, IMR, particularly Nur Syamimi, H.S., Muhammad Irfan Norman, F.R., Faiz, A., Thohir, H., Nuradila, N., Mohd Irwan, S., Muhd Nizam, M.N. and Rubaaini, S. for their technical assistance in collecting and identifying the mosquitoes.

Conflicts of Interest: The authors declare no conflict of interest. The funders had no role in the design of the study; in the collection, analyses, or interpretation of data; in the writing of the manuscript, or in the decision to publish the results.

\section{References}

1. Ministry of Health Malaysia. 2019. Available online: http://idengue.remotesensing.gov.my/idengue/index. php (accessed on 9 August 2020).

2. Loke, S.R.; Ann, A.T.W.; Nazni, W.A.; Lee, H.L.; Sofian Azirun, M. Insecticide susceptibility status of field-collected Aedes (Stegomyia) aegypti (L.) at a dengue endemic site in Shah Alam, Selangor, Malaysia. Southeast Asian J. Trop. Med. Public Health 2012, 43, 34-47.

3. Wan-Norafikah, O.; Nazni, W.A.; Lee, H.; Zainol-Ariffin, P.; Sofian-Azirun, M. Permethrin resistance in Aedes aegypti (Linnaeus) collected from Kuala Lumpur, Malaysia. J. Asia Pac. Entomol. 2010, 13, 175-182. [CrossRef]

4. Ishak, I.H.; Jaal, Z.; Ranson, H.; Wondji, C.S. Contrasting patterns of insecticide resistance and knockdown resistance $(k d r)$ in the dengue vectors Aedes aegypti and Aedes albopictus from Malaysia. Parasites Vectors 2015, 8, 1-13. [CrossRef] [PubMed]

5. Hidayati, H.; Sofian Azirun, M.; Nazni, W.A.; Lee, H.L. Insecticide resistance development in Culex quinquefasciatus (Say), Aedes aegypti (L.) and Aedes albopictus (Skuse) larvae against malathion, permethrin and temephos. Trop. Biomed. 2005, 22, 45-52.

6. WHO. Chemistry and Specifications on Insecticides: Sixteenth Report of the WHO Expert Committee on Vector Biology and Control; WHO Technical Report Series 899; WHO: Geneva, Switzerland, 2001.

7. Chen, C.D.; Nazni, W.A.; Lee, H.L.; Sofian Azirun, M. Susceptibility of Aedes aegypti and Aedes albopictus to temephos in four study sites in Kuala Lumpur City Center and Selangor State, Malaysia. Trop. Biomed. 2005, 22, 207-216. [PubMed]

8. Nazni, W.A.; Lee, H.L.; Azahari, A.H. Adult and larval insecticide susceptibility status of Culex quinquefasciatus (Say) mosquitoes in Kuala Lumpur. Trop. Biomed. 2005, 22, 63-68.

9. Rohani, A.; Ismail, S.; Malinda, M.; Anuar, I.; Mohd Mazlan, I.; Salmah Maszaitun, M. Aedes larval population dynamics and risk for dengue epidemics in Malaysia. Trop. Biomed. 2011, 28, 237-248. 
10. El-Garj, F.M.A.; Wajidi, M.F.F.; Avicor, S.W. Allelic variants of cytochrome P450 monooxygenases: Constitutive and insecticide-mediated expression in a Malaysian strain of the dengue vector, Aedes aegypti (Diptera: Culicidae). Eur. J. Entomol. 2016, 113, 507-515. [CrossRef]

11. Ishak, I.H.; Kamgang, B.; Ibrahim, S.S.; Riveron, J.M.; Irving, H.; Wondji, C.S. Pyrethroid resistance in Malaysian populations of dengue vector Aedes aegypti is mediated by CYP9 family of cytochrome P450 genes. PLoS Negl. Trop. Dis. 2017, 11,1-20. [CrossRef]

12. Ishak, I.H.; Riveron, J.M.; Ibrahim, S.S.; Stott, R.; Longbottom, J.; Irving, H.; Wondji, C.S. The Cytochrome P450 gene CYP6P12 confers pyrethroid resistance in $k d r$-free Malaysian populations of the dengue vector Aedes albopictus. Sci. Rep. 2016, 6, 24707. [CrossRef]

13. Du, Y.; Nomura, Y.; Satar, G.; Hu, Z.; Nauen, R.; He, S.Y.; Zhorov, B.S.; Dong, K. Molecular evidence for dual pyrethroid-receptor sites on a mosquito sodium channel. Proc. Natl. Acad. Sci. USA 2013, 110, 11785-11790. [CrossRef] [PubMed]

14. Wuliandari, J.; Lee, S.; White, V.; Tantowijoyo, W.; Hoffmann, A.; Endersby-Harshman, N. Association between three mutations, F1565C, V1023G and S996P, in the voltage-sensitive sodium channel gene and knockdown resistance in Aedes aegypti from Yogyakarta, Indonesia. Insects 2015, 6, 658-685. [CrossRef] [PubMed]

15. Sayono, S.; Hidayati, A.P.N.; Fahri, S.; Sumanto, D.; Dharmana, E.; Hadisaputro, S.; Asih, P.B.S.; Syafruddin, D. Distribution of Voltage-Gated Sodium Channel (Nav) alleles among the Aedes aegypti populations in Central Java Province and its association with resistance to pyrethroid insecticides. PLoS ONE 2016, 11, e0150577. [CrossRef]

16. Du, Y.; Nomura, Y.; Zhorov, B.S.; Dong, K. Sodium channel mutations and pyrethroid resistance in Aedes aegypti. Insects 2016, 7, 60. [CrossRef] [PubMed]

17. Yanola, J.; Somboon, P.; Walton, C.; Nachaiwieng, W.; Somwang, P.; Prapanthadara, L.-A. High-throughput assays for detection of the F1534C mutation in the voltage-gated sodium channel gene in permethrin-resistant Aedes aegypti and the distribution of this mutation throughout Thailand. Trop. Med. Int. Health TM IH 2011, 16, 501-509. [CrossRef]

18. Kawada, H.; Higa, Y.; Komagata, O.; Kasai, S.; Tomita, T.; Nguyen Thi, Y.; Luu Lee, L.; Sánchez, R.A.P.; Takagi, M. Widespread distribution of a newly found point mutation in Voltage-Gated Sodium Channel in pyrethroid-resistant Aedes aegypti populations in Vietnam. PLoS Negl. Trop. Dis. 2009, 3, e527. [CrossRef] [PubMed]

19. Harris, A.F.; Rajatileka, S.; Ranson, H. Pyrethroid resistance in Aedes aegypti from Grand Cayman. Am. J. Trop. Med. Hyg. 2010, 83, 277-284. [CrossRef]

20. Linss, B.; Gerlinde, J.; Brito, L.P.; Azambuja Garcia, G.; Saori Araki, A.; Vieira Bruno, R.; Pereira Lima, J.B.; Valle, D.; Martins, A.J. Distribution and dissemination of the Val1016Ile and Phe1534Cys $k d r$ mutations in Aedes aegypti Brazilian natural populations. Parasites Vectors 2014, 7, 1-21. [CrossRef]

21. Li, C.-X.; Kaufman, P.E.; Xue, R.-D.; Zhao, M.-H.; Wang, G.; Yan, T.; Guo, X.-X.; Zhang, Y.-M.; Dong, Y.-D.; Xing, D.; et al. Relationship between insecticide resistance and $k d r$ mutations in the dengue vector Aedes aegypti in Southern China. Parasites Vectors 2015. [CrossRef]

22. Endersby-Harshman, N.M.; Schmidt, T.L.; Chung, J.; van Rooyen, A.; Weeks, A.R.; Hoffmann, A.A. Heterogeneous genetic invasions of three insecticide resistance mutations in Indo-Pacific populations of Aedes aegypti (L.). Mol. Ecol. 2020. [CrossRef]

23. Kawada, H.; Oo, S.Z.M.; Thaung, S.; Kawashima, E.; Maung, Y.N.M.; Thu, H.M.; Thant, K.Z.; Minakawa, N. Co-occurrence of point mutations in the voltage-gated sodium channel of pyrethroid-resistant Aedes aegypti populations in Myanmar. PLoS Negl. Trop Dis. 2014, 8, e3032. [CrossRef] [PubMed]

24. Al Nazawi, A.M.; Aqili, J.; Alzahrani, M.; McCall, P.J.; Weetman, D. Combined target site ( $k d r)$ mutations play a primary role in highly pyrethroid resistant phenotypes of Aedes aegypti from Saudi Arabia. Parasites Vectors 2017, 10, 161. [CrossRef] [PubMed]

25. Nazni, W.A.; Hoffmann, A.A.; NoorAfizah, A.; Cheong, Y.L.; Mancini, M.V.; Golding, N.; Kamarul, G.M.R.; Arif, M.A.K.; Thohir, H.; NurSyamimi, H.; et al. Establishment of Wolbachia strain wAlbB in Malaysian populations of Aedes aegypti for dengue control. Curr. Biol. 2019, 29, 4241-4248. [CrossRef] [PubMed]

26. Ant, T.H.; Herd, C.S.; Geoghegan, V.; Hoffmann, A.A.; Sinkins, S.P. The Wolbachia strain wAu provides highly efficient virus transmission blocking in Aedes aegypti. PLoS Pathog. 2018, 14, e1006815. [CrossRef] [PubMed] 
27. Yeap, H.L.; Mee, P.; Walker, T.; Weeks, A.R.; O’Neill, S.L.; Johnson, P.; Ritchie, S.A.; Richardson, K.M.; Doig, C.; Endersby, N.M.; et al. Dynamics of the "popcorn" Wolbachia infection in outbred Aedes aegypti informs prospects for mosquito vector control. Genetics 2011, 187, 583-595. [CrossRef]

28. Hoffmann, A.A.; Montgomery, B.L.; Popovici, J.; Iturbe-Ormaetxe, I.; Johnson, P.H.; Muzzi, F.; Greenfield, M.; Durkan, M.; Leong, Y.S.; Dong, Y.; et al. Successful establishment of Wolbachia in Aedes populations to suppress dengue transmission. Nature 2011, 476, 454-457. Available online: http:/www.nature.com/ nature/journal/v476/n7361/abs/nature10356.html\#supplementary-information (accessed on 9 August 2020). [CrossRef]

29. Endersby, N.M.; Hoffmann, A.A. Effect of Wolbachia on insecticide susceptibility in lines of Aedes aegypti. Bull. Entomol. Res. 2013, 103, 269-277. [CrossRef]

30. Garcia, G.A.; Hoffmann, A.A.; Maciel-de-Freitas, R.; Villela, D.A.M. Aedes aegypti insecticide resistance underlies the success (and failure) of Wolbachia population replacement. Sci. Rep. 2020, 10, 1-9. [CrossRef]

31. Garcia, G.d.A.; Sylvestre, G.; Aguiar, R.; da Costa, G.B.; Martins, A.J.; Lima, J.B.P.; Petersen, M.T.; Lourenço-de-Oliveira, R.; Shadbolt, M.F.; Rašić, G.; et al. Matching the genetics of released and local Aedes aegypti populations is critical to assure Wolbachia invasion. PLoS Negl. Trop. Dis. 2019, 13, e0007023. [CrossRef]

32. Tee, A.S.; Daud, A.R.; Alias, M.; Lee, H.L.; Tham, A.S. Guidelines on the Use of Ovitraps for Aedes Surveillance. Kuala Lumpur: Vector Control. Unit Vector Borne Disease Section; Ministry of Health Malaysia: Putrajaya, Malaysia, 1997.

33. Noor Afizah, A.; Mahirah, M.; Azahari, A.; Khairul Asuad, M.; Nazni, W.; Lee, H. Absence of Aedes aegypti (L.) on an ecological island: Competitive Exclusion? Southeast Asian J. Trop. Med. Public Health 2015, 46, 850-856.

34. WHO. Instructions for Determining the Susceptibility or Resistance of Mosquito Larvae to Insecticides; World Health Organization: Geneva, Switzerland, 1981; Available online: http://www.who.int/iris/handle/10665/69615 (accessed on 9 August 2020).

35. WHO. Monitoring and Managing Insecticide Resistance in Aedes Mosquito Populations: Interim Guidance for Entomologists; World Health Organization: Geneva, Switzerland, 2016; Available online: http://www.who.int/ iris/handle/10665/204588 (accessed on 9 August 2020).

36. Kasai, S.; Komagata, O.; Itokawa, K.; Shono, T.; Ng, L.C.; Kobayashi, M.; Tomita, T. Mechanisms of pyrethroid resistance in the dengue mosquito vector, Aedes aegypti: Target site insensitivity, penetration, and metabolism. PLoS Negl. Trop. Dis. 2014, 8, e2948. [CrossRef] [PubMed]

37. Kasai, S.; Ng, L.C.; Lam-Phua, S.G.; Tang, C.S.; Itokawa, K.; Komagata, O.; Kobayashi, M.; Tomita, T. First detection of a putative knockdown resistance gene in major mosquito vector, Aedes albopictus. Jpn. J. Infect. Dis. 2011, 64, 217-221. [PubMed]

38. Endersby-Harshman, N.M.; Wuliandari, J.R.; Harshman, L.G.; Frohn, V.; Johnson, B.J.; Ritchie, S.A.; Hoffmann, A.A. Pyrethroid susceptibility has been maintained in the dengue vector, Aedes aegypti (Diptera: Culicidae), in Queensland, Australia. J. Med. Entomol. 2017, 54, 1649-1658. [CrossRef] [PubMed]

39. Walker, T.; Johnson, P.H.; Moreira, L.A.; Iturbe-Ormaetxe, I.; Frentiu, F.D.; McMeniman, C.J.; Leong, Y.S.; Dong, Y.; Axford, J.; Kriesner, P.; et al. The wMel Wolbachia strain blocks dengue and invades caged Aedes aegypti populations. Nature 2011, 476, 450-453. Available online: http://www.nature.com/nature/journal/ v476/n7361/abs/nature10355.html\#supplementary-information (accessed on 9 August 2020). [CrossRef] [PubMed]

40. Smith, L.B.; Kasai, S.; Scott, J.G. Pyrethroid resistance in Aedes aegypti and Aedes albopictus: Important mosquito vectors of human diseases. Pestic. Biochem. Physiol. 2016, 133, 1-12. [CrossRef]

41. Su, X.; Guo, Y.; Deng, J.; Xu, J.; Zhou, G.; Zhou, T.; Li, Y.; Zhong, D.; Kong, L.; Wang, X.; et al. Fast emerging insecticide resistance in Aedes albopictus in Guangzhou, China: Alarm to the dengue epidemic. PLoS Negl. Trop. Dis. 2019, 13, e0007665. [CrossRef]

42. Zhou, X.; Li, M.; Liu, N.; Qiu, X.; Tong, Y.; Yang, C.; Zeng, X. Knockdown resistance ( $k d r)$ mutations within seventeen field populations of Aedes albopictus from Beijing China: First report of a novel V1016G mutation and evolutionary origins of kdr haplotypes. Parasites Vectors 2019, 12, 180. [CrossRef]

43. Marcombe, S.; Farajollahi, A.; Healy, S.P.; Clark, G.G.; Fonseca, D.M. Insecticide resistance status of United States populations of Aedes albopictus and mechanisms involved. PLoS ONE 2014, 9, e101992. [CrossRef] 
44. Xu, J.; Bonizzoni, M.; Zhong, D.; Zhou, G.; Cai, S.; Li, Y.; Wang, X.; Lo, E.; Lee, R.; Sheen, R.; et al. Multi-country survey revealed prevalent and novel F1534S mutation in Voltage-Gated Sodium Channel (VGSC) gene in Aedes albopictus. PLoS Negl. Trop. Dis. 2016, 10, e0004696. [CrossRef]

45. Yan, R.; Zhou, Q.; Xu, Z.; Zhu, G.; Dong, K.; Zhorov, B.S.; Chen, M. Three sodium channel mutations from Aedes albopictus confer resistance to Type I, but not Type II pyrethroids. Insect Biochem. Mol. Biol. 2020. [CrossRef]

46. Li, Y.; Xu, J.; Zhong, D.; Zhang, H.; Yang, W.; Zhou, G.; Su, X.; Wu, Y.; Wu, K.; Cai, S.; et al. Evidence for multiple-insecticide resistance in urban Aedes albopictus populations in southern China. Parasites Vectors 2018, 11, 4. [CrossRef] [PubMed]

47. Rasli, R.; Lee, H.L.; Wasi Ahmad, N.; Fikri, S.F.F.; Ali, R.; Muhamed, K.A.; Hadi, A.A.; Liu, Q.Y.; Meng, F.X. Susceptibility status and resistance mechanisms in permethrin-selected, laboratory susceptible and field-collected Aedes aegypti from Malaysia. Insects 2018, 9, 43. [CrossRef] [PubMed]

48. Platt, N.; Kwiatkowska, R.M.; Irving, H.; Diabaté, A.; Dabire, R.; Wondji, C.S. Target-site resistance mutations ( $k d r$ and $R D L$ ), but not metabolic resistance, negatively impact male mating competiveness in the malaria vector Anopheles gambiae. Heredity 2015, 115, 243-252. [CrossRef] [PubMed]

(C) 2020 by the authors. Licensee MDPI, Basel, Switzerland. This article is an open access article distributed under the terms and conditions of the Creative Commons Attribution (CC BY) license (http://creativecommons.org/licenses/by/4.0/). 